\title{
Componente del Internet de las cosas para detectar patrones de deambulaje en pacientes con demencia
}

\author{
Hector Villarreal ${ }^{1}$, Mayra Lizarraga ${ }^{1}$, Arnoldo Diaz-Ramírez $^{1}$, \\ Verónica Quintero Rosas ${ }^{1}$, Juan Pablo García-Vázquez ${ }^{2}$ \\ 1 Tecnológico Nacional de México/IT de Mexicali, \\ Departamento de Sistemas y Computación, México \\ \{hevr93, mayra.lizarraga] @gmail.com, \\ \{adiaz, veronicaquintero\}@itmexicali.edu.mx \\ ${ }^{2}$ Universidad Autónoma de Baja California, Facultad de Ingeniería, \\ México \\ pablo.garcia@uabc.edu.mx
}

Resumen. La demencia es un problema de salud que afecta la vida de millones de personas en todo el mundo. Esta enfermedad, además de ser la causa de muerte de muchas personas, provoca que los enfermos sean incapaces de realizar actividades de índole cotidiano. Adicionalmente, el deambulaje es uno de los síntomas de la demencia. Cuando un enfermo deambula hace desplazamientos o caminatas sin supervisión, con la esperanza de encontrar algún lugar o persona que le resulte familiar. Detectar el nivel de avance de la enfermedad de demencia no es una tarea sencilla. Debido a que se trata de padecimientos causados por daños cerebrales, una manera de conocer el estado de la enfermedad es a través de pruebas costosas, tales como tomografías o resonancias magnéticas. Sin embargo, estas pruebas pueden provocar demasiado estrés en los pacientes, lo que puede dificultar su aplicación. Por tal motivo es importante desarrollar métodos alternativos y no invasivos para este fin. Recientemente se ha detectado que existe una relación entre los patrones de deambulaje y el nivel del avance de la demencia. En este artículo se presenta un componente para la detección de patrones de deambulaje de pacientes afectados con demencia. El componente propuesto, desarrollado con base en el paradigma del Internet de las Cosas, no es invasivo ni requiere de infraestructura previamente instalada en los edificios en los que será utilizado. La evaluación del sistema propuesta mostraron una alta tasa de detección.

Palabras clave: demencia, Internet de las cosas, deambulaje.

\section{Component of the Internet of Things for Detection of Wandering Patterns in Patients with Dementia}

Abstract. Dementia is a health problem that affects millions of persons around the world. This disease, which is one of the main death causes 
of the elderly, does not allow them to perform every day activities. Additionally, wandering is a common symptom of people affected by dementia. When a patient wanders, she walks without supervision seeking for a familiar place or person. Detecting the level of dementia is not an easy task. Since dementia is caused by brain damage, on way to detect it is trough the use of invasive techniques, such as tomographies or magnetic resonance, which may cause a lot of stress in patients. Recently, a relationship between the level of dementia and wandering patterns has been found. In this paper, a component based on the Internet of Things paradigm to detect wandering patterns of people affected by dementia is introduced. The proposed component is not invasive and does not require previously installed infrastructure. The evaluation of the component showed a very good detection rate.

Keywords: dementia, Internet of things, wandering.

\section{Introducción}

La demencia es un problema de salud que afecta la vida de millones de personas en todo el mundo. Debido a su impacto, ha recibido mucha atención de especialistas de diversas áreas del conocimiento [18]. La demencia puede afectar completamente el estilo de vida de quienes la padecen y de los que los rodean, llegando a cambiar la vida de las personas, familias e incluso comunidades. Esta enfermedad, además de ser la causa de muerte de muchas personas, provoca que los enfermos sean incapaces de realizar actividades de índole cotidiano.

La demencia no es una enfermedad que se pueda predecir o identificar con un gran grado de exactitud, ya que tiende a no ser específica; es decir, puede variar de paciente en paciente, e incluso los síntomas pueden ser diferentes en cada uno de los afectados. La demencia es el nombre genérico que recibe una amplia gama de enfermedades que afectan las funciones cognitivas. Cada tipo de demencia afecta una región particular del cerebro [4]. Por ejemplo, la enfermedad del Alzheimer provoca que los niveles de algunas proteínas dentro y fuera de las células cerebrales aumenten [8]. Como consecuencia de esto, las células de la región afectada no pueden mantenerse saludables y tienen problemas para comunicarse. Esta enfermedad ataca principalmente a la región cerebral del hipocampo, el cual es el encargado del aprendizaje y la memoria. Por tal motivo, la pérdida de memoria es una de los principales síntomas de la enfermedad del Alzheimer [2].

Las estadísticas indican que año con año aumentan el número de casos de personas afectadas con demencia. Se estimó que para el año 2017, 5.5 millones de estadounidenses mayores a 65 años sufrirían algún tipo de demencia, de los cuales 200,000 serían personas menores de 65 años [5]. En México, en el año 2009 se registraron 1,200 casos de muertes por algún tipo de demencia. Además, se registraron un poco mas de 142, 000 casos confirmados en ese año [17]. 
La demencia es una enfermedad que no tiene cura, que es progresiva y que afecta no solo la calidad de vida del paciente sino de quienes lo cuidan. El tratamiento para este padecimiento varía de acuerdo al grado de avance de la enfermedad. Un diagnóstico erróneo puede provocar situaciones de riesgo tanto en pacientes como en cuidadores. Un paciente puede cambiar de etapa en el avance de su enfermedad y presentar nuevos comportamientos. Por ejemplo, salir de su casa sin supervisión, extraviarse y poner en riesgo su integridad física o su vida. Por tal motivo, poder saber con anticipación si un paciente corre este riesgo es de vital importancia.

Detectar el nivel de avance de la enfermedad de demencia no es una tarea sencilla. Debido a que se trata de padecimientos causados por daños cerebrales, una manera de conocer el estado de la enfermedad es a través de pruebas costosas, tales como encefalogramas, tomografías o resonancias magnéticas. Sin embargo, estas pruebas pueden provocar demasiado estrés en los pacientes, lo que puede dificultar su aplicación. Por tal motivo es importante desarrollar métodos alternativos y no invasivos para este fin.

El deambulaje es uno de los síntomas de la demencia. Cuando un enfermo deambula hace desplazamientos o caminatas sin supervisión, con la esperanza de encontrar algún lugar o persona que le resulte familiar. Este fenómeno se lleva a cabo a pesar de que el paciente esté en su propio hogar o rodeado de personas conocidas [3]. Es común que mientras una persona deambula sea incapaz de reconocer el entorno que lo rodea, debido a los problemas de memoria y de concentración producidos por la demencia. Como consecuencia del deambulaje los pacientes pueden sufrir accidentes como caídas, golpes, torceduras, etc. Los episodios de deambulaje tienen una duración máxima de de 5.41 minutos [1]. Por otra parte, se considera que un episodio concluye cuando la persona pasa mas de 15 segundos detenido en el mismo sitio [10].

Martino-Saltzman et al. en [15] identificaron que existe una relación entre el nivel de deterioro de la persona afectada por la demencia y el tipo de trayectoria que presenta al caminar, particularmente cuando deambula. En el desarrollo de los experimentos presentados en [15] se identificaron tres grupos de personas, clasificados por el tipo de avance de la demencia en cada individuo: nula/leve, moderada y avanzada. Durante los experimentos se registraron mas de 5,500 episodios de deambulaje de 40 residentes de 130 estancias geriátricas de la ciudad de Atlanta, Georgia, Estados Unidos. De los 40 residentes, 24 fueron identificados con tendencias a deambular, llamados también errantes debido a la naturaleza imprecisa o errónea de sus desplazamientos. Los 16 pacientes restantes fueron identificados como personas sin tendencias a deambular o no errantes.

Con base en la observación de los patrones de deambulaje, estos fueron clasificados en cuatro grupos: directo (direct), rítmico (pacing), cíclico (lapping) y aleatorio (random). El primero representa un desplazamiento de un punto a otro sin desviación. El segundo, denominado rítmico, representa un desplazamiento repetitivo, de un punto a otro y de regreso, dentro de una área delimitada. En el patrón cíclico la persona se desplaza en movimientos semi-circulares, a menudo en repetidas ocasiones. En el movimiento aleatorio el paciente se 
desplaza a diferentes puntos, sin una área delimitada y sin repeticiones. El patrón directo es considerado como un desplazamiento eficiente y síntoma de salud. En contraste, los patrones rítmico, cíclico y aleatorio son considerados desplazamientos ineficientes y relacionados con deterioro cognitivo. En el Cuadro 1 se muestran los resultados de los experimentos realizados en [15] de pacientes errantes y no errantes, clasificados por patrón de deambulaje. Puede observarse que la mayoría de los episodios de deambulaje de personas no errantes fueron eficientes $(94 \%)$. Esta tasa disminuyó en los pacientes no errantes, siendo el patrón cíclico el mas común de los episodios no eficientes $(17.3 \%)$.

Tabla 1. Comportamientos presentados por los pacientes en contraste errantes y no errantes [15].

\begin{tabular}{ccccc}
\hline \multirow{2}{*}{ TIPO DE PACIENTE } & \multicolumn{4}{c}{ PATRONES DE MOVIMIENTOS } \\
\cline { 2 - 5 } & Directo & Aleatorio & Ritmico & Cíclico \\
\hline Errante & $81.3 \%$ & $1 \%$ & $0.1 \%$ & $17.3 \%$ \\
\hline No Errante & $94 \%$ & $0.4 \%$ & $1.3 \%$ & $4.3 \%$ \\
\hline
\end{tabular}

De los 40 residentes participantes en el estudio, a 32 de ellos se les diagnosticó algún tipo de demencia: ligera, moderada o severa. El Cuadro 1 muestra la clasificación de los experimentos realizados en [15] de los episodios de deambulaje de los pacientes afectados por demencia. Los episodios fueron clasificados por patrón de deambulaje. Puede observarse que a mayor daño cognitivo es mayor la tasa de desplazamientos ineficientes.

Tabla 2. Patrón de deambulaje clasificado por avance de la enfermedad [15].

\begin{tabular}{cccc}
\hline \multirow{2}{*}{ CARACTERISTICAS } & \multicolumn{4}{c}{ NIVEL DE DEMENCIA } \\
\cline { 2 - 4 } & Ligero $/$ Nulo $(N=12)$ & Moderado $(N=11)$ & Severa $(N=9)$ \\
\hline Directa & $94.7 \%$ & $92.5 \%$ & $72 \%$ \\
\hline Aleatoria & $0.2 \%$ & $1 \%$ & $1.7 \%$ \\
\hline Rítmica & $1.5 \%$ & $0.0 \%$ & $0.3 \%$ \\
\hline Cíclica & $3.6 \%$ & $6.5 \%$ & $26 \%$ \\
\hline
\end{tabular}

Los resultados presentados en [15] identifican una relación entre el grado de avance de la demencia y los patrones de deambulaje. Es importante señalar que la identificación de los patrones de deambulaje en [15] se hicieron a través de grabaciones de video y observación. Este es un proceso costoso y lento. Por tal motivo se destaca la importancia de contar con un sistema alterno para su detección. En este artículo se presenta un componente para la detección de 
patrones de deambulaje de pacientes afectados con demencia. El componente propuesto, desarrollado con base en el paradigma del Internet de las Cosas, no es invasivo ni requiere de infraestructura previamente instalada en los edificios en los que será utilizado. La evaluación del sistema propuesta mostraron una alta tasa de detección.

El resto del documento está organizado de la siguiente manera. La Sección 2 presenta algunos de los trabajos relacionados. En la Sección 3 se discute la arquitectura del componente propuesto. La Sección 4 presenta los resultados de su evaluación. Finalmente, en la Sección 5 está dedicada a las conclusiones y el trabajo futuro.

\section{Trabajos relacionados}

Existen varias propuestas para monitorear actividades de personas afectadas con demencia. En esta sección se presentan algunas de las mas importantes.

El proyecto iWander es un sistema para la monitorización de personas con demencia que pretende ser una opción económica y sencilla de implementar para el apoyo a los cuidadores. El sistema está diseñado para hacer uso de los sensores existentes en un dispositivo móvil, entre los que destacan el GPS [13]. Este sistema se encarga de definir un espacio seguro donde interactúa el usuario, como es el hogar de la persona. Y si el paciente sale de esta zona segura, el programa ejecuta un algoritmo para definir si se trata de un episodio de deambulaje o no [21].

La implementación del algoritmo se divide en 3 fases. La primera se denomina pre-instalación, que consiste en la recopilación de datos sobre el usuario, además de la creación de las variables necesarias para la funcionalidad del sistema. Las relaciones y estadísticas entre las variables son las únicas entradas de datos. La segunda es denominada configuración. En esta fase se configura el dispositivo para un usuario en particular. Para ello se solicita información como la edad y el nivel de demencia. Otros datos necesarios para el algoritmo se obtienen de manera automática por el sistema. La última fase es la de aprendizaje extendido. En esta fase se analizan los hábitos del usuario, se definen las zonas seguras e identifican los episodios de deambulaje. Gracias a esta fase, el sistema aprende constantemente. A mayor sea el uso, el sistema es más preciso y eficaz.

Si el paciente sale de la zona segura, se considera que la persona se encuentra en un episodio de demencia, por lo que se toman medidas antes de mandar una alerta. En primer lugar, una notificación le solicita a los pacientes que indiquen si están bien. En caso de ser positivo, el proceso de alerta se detendrá, reduciendo así los falsos positivos. Si el paciente no responde, se deduce que él o ella no está bien y puede perderse. Las coordenadas de GPS se utilizan con las herramientas de navegación de Google Maps [12], para darles instrucciones sobre la zona segura. Si no se realiza ningún progreso después de un período de tiempo determinado, se envía una alerta a los cuidadores.

iSenior [19] es un sistema ciber-físico que soporta funciones como monitorización, alerta y solicitudes de asistencia. Este sistema es una herramienta para el 
apoyo a personas mayores. El sistema proporciona apoyo como monitorización y alertas en parámetros como latidos del corazón y nivel de actividad, así como detección de situaciones potencialmente peligrosas. Adicionalmente, proporciona funciones para supervisar las condiciones de salud, que abarcan la recopilación y el almacenamiento de datos, la administración de alarmas, la localización, el acceso remoto, varios perfiles de usuario y la configuración automática.

El sistema cuenta con la capacidad de comunicarse con una unidad central a través de GSM/GPRS [16] [7] o a través de una red inalámbrica de sensores o WSN [11]. Cuenta con una arquitectura especializada, a través de múltiples sensores que se encargan de recolectar información del paciente, quedando a disposición de los cuidadores, los cuales pueden acceder desde cualquier equipo o dispositivo móvil. Además, cuenta con la capacidad de alertar a los cuidadores en caso de que alguno de ellos deje su cuarto o incluso el edificio. Además, es capaz de identificar la dirección en la que se dirige el paciente, pudiendo así el cuidador conocer la ubicación actual evitando accidentes durante el proceso.

En [14], Lin et al. propusieron un algoritmo denominado $\theta_{-} W D$, para la detección de trayectorias circulares o cíclicas en pacientes afectados con demencia. El algoritmo utiliza el sensor GPS de los dispositivos móviles para identificar el tipo de trayectoria. Una limitante de esta propuesta es que tan solo funciona en exteriores.

En [22] se propone un algoritmo para la detección de los patrones de deambulaje identificados por Martino-Saltzman et al. [15]. Utilizando la potencia de la señal de puntos de acceso WiFi previamente instalados en el edificio, el algoritmo determina la trayectoria del paciente. Para un cálculo sencillo, se supone un tiempo de viaje fijo, entre dos ubicaciones conocidas. Cada episodio de deambulaje (errante y no deseable) consiste en uno o varios movimientos consecutivos. El punto de inicio de un episodio es el punto final del episodio anterior. El algoritmo se inicializa para comenzar en el punto de inicio de un episodio.

Hasta donde los autores conocen, no existe un método para la detección de los patrones de deambulaje de los pacientes con demencia que funcione en interiores y que no requiera infraestructura previamente instalada en el edificio. En la siguiente sección se presenta un componente con esas características.

\section{Arquitectura del componente propuesto}

En este artículo se propone un componente capaz de detectar y categorizar los patrones de deambulaje de pacientes con demencia. El componente se basa en el paradigma del Internet de las Cosas, en el que sistemas embebidos, equipados con sensores, son colocados en cosas o personas para que de manera ubicua monitoricen y detecten eventos de interés, y a través de tecnologías de comunicación inalámbrica permitan que usuarios o sistemas informáticos reciban la información recolectada y puedan actuar en consecuencia [6]. El componente propuesto no requiere infraestructura previamente instalada en el hogar o residencia geriátrica en la que habita el paciente. La arquitectura general 
del componente propuesto se presenta en la Fig. 1. Como puede observarse, el componente consta de cinco módulos, que se describen a continuación.

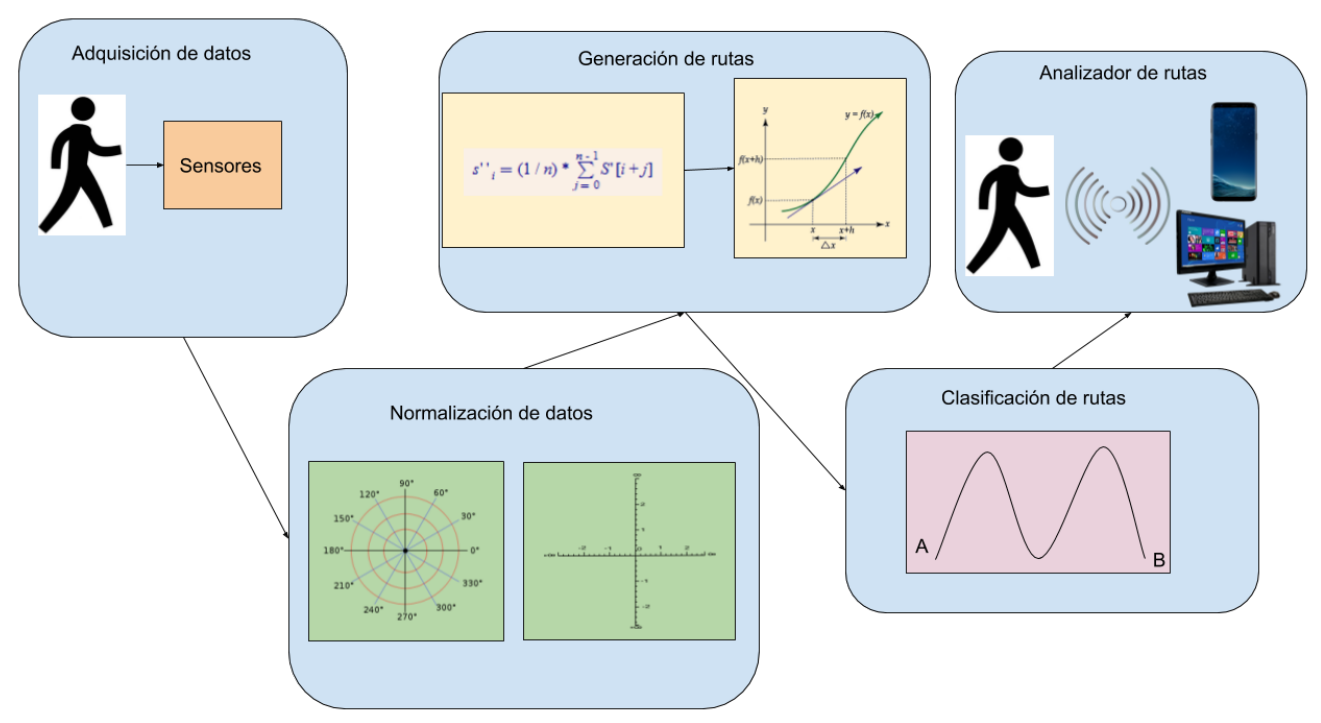

Fig. 1. Arquitectura del sistema.

\subsection{Módulo de adquisición de datos}

El módulo de adquisición de datos tiene como objetivo tomar mediciones de la aceleración y la dirección de movimiento del paciente, además de la intensidad campo magnético terrestre, con el propósito de tener una geolocalización del sujeto. Para este fin se utilizan sensores como el magnetómetro, el giroscopio y el acelerómetro, en un dispositivo adherido a la persona. Los sensores, a pesar de contar con una gran precisión en la actualidad, en ocasiones registran mediciones erróneas debido al ruido externo o fallos en el mismo sensor. Para tratar dichos errores es común utilizar algún filtro, que es un algoritmo de procesamiento digital de señales. Existen múltiples tipos de filtros, que ayudan con diferentes propósitos. El módulo de adquisición de datos utiliza un filtro paso-bajo para reducir el efecto de las mediciones erróneas de los sensores, tal y como el utilizado en sistema propuesto en [9], que permite la identificación y trazado de rutas de personas en interiores, sin necesidad de infraestructura previamente instalada.

\subsection{Módulo de normalización de datos}

Con las mediciones realizadas por los sensores del dispositivo, se obtienen valores del ángulo y la distancia del movimiento del paciente. Utilizando estos 
datos como coordenadas polares y aplicando un par de funciones matemáticas, se obtienen coordenadas rectangulares del sujeto, pudiendo identificar las posiciones del mismo mientras se desplaza.

Teniendo un vector $S$ conformado por $n$ tuplas de datos obtenidos mediante los sensores, se llamará $\alpha$ a los datos que correspondan a la dirección de un paso y $d$ a los datos que correspondan a la distancia del paso, donde $i$ corresponde a la $i$-esima medición. Por lo tanto, se tiene que

$$
S=\left\{s_{i}=\left(\alpha_{i}, d_{i}\right)|1 \leq i \leq n| i, n \in \mathbb{N}\right\}
$$

Definiendo estos datos como coordenadas polares, por cuestión de simplicidad, se llevará a cabo una transformación de coordenadas polares a coordenadas rectangulares:

$$
\begin{aligned}
& x_{i}=d_{i} \times \sin \left(\alpha_{i}\right)|1 \leq i \leq n| i, n \in \mathbb{N}, \\
& y_{i}=d_{i} \times \cos \left(\alpha_{i}\right)|1 \leq i \leq n| i, n \in \mathbb{N},
\end{aligned}
$$

donde $x$ son coordenadas en el eje horizontal de un plano y $y$ son coordenadas en el eje vertical de un plano, $i$ es el $i$-esimo dato de las mediciones registradas por los sensores. Tomando ambos valores, se pueden definir como coordenadas rectangulares en un plano, definiendo entonces que $s_{i}^{\prime}=\left(x_{i}, y_{i}\right)$, por lo tanto

$$
S^{\prime}=\left\{s_{i}^{\prime}=\left(x_{i}, y_{i}\right)|1 \leq i \leq n| i, n \in \mathbb{N}\right\},
$$

donde $S^{\prime}$ representa un vector de coordenadas rectangulares.

\subsection{Módulo de generación de rutas}

Si las distancias recorridas por una persona son mayores a veinte metros, el módulo de adquisición de datos es capaz de detectar las rutas con un aceptable grado de precisión. Sin embargo, si las distancias recorridas son de menor longitud, como es común en desplazamientos de las personas afectadas por demencia, es necesario utilizar otro filtro. En el módulo de generación de rutas se utiliza el filtro de movimiento promedio [20], debido a que este filtro cuenta con la capacidad de ajustarse a una gráfica modelo, por lo que la pérdida de información es mínima y su implementación es sencilla. Para ello se aplica la función de suavizado:

$$
s_{i}^{\prime \prime}=(1 / n) \times \sum_{j=0}^{n-1} S^{\prime}[i+j] .
$$

En esta función $n$ es el número de mediciones totales hechas por los sensores, en donde, $i$ es la $i$-esima columna de la matriz de mediciones y $j$ es la $j$-esima línea de la matriz. En la matriz de mediciones las columnas son los valores de los ejes de un plano y los renglones son cada una de las mediciones realizadas. En otras palabras, lo que se realiza es promediar un número de puntos de una señal de entrada para producir los puntos de una señal de salida. 
Por lo tanto, se creará un nuevo vector de datos que llamaremos $S^{\prime \prime}$, donde $s_{i}^{\prime \prime}=\left(x_{i}^{\prime \prime}, y_{i}^{\prime \prime}\right)$, donde $x^{\prime \prime}$ y $y^{\prime \prime}$ son las coordenadas de salida después de la aplicación de la función de suavizado, por lo tanto, es correcto decir que

$$
S^{\prime \prime}=\left\{s_{i}^{\prime \prime}=\left(x_{i}^{\prime \prime}, y_{i}^{\prime \prime}\right)|1 \leq i \leq n| i, n \in \mathbb{N}\right\}
$$

El procedimiento de identificaciones de diferencias en los datos de los tres tipos de movimientos (directo, rítmico y cíclico) definidos por [15], se realizó por medio de la pendiente de las rectas que representan las trayectorias de cada paso los pacientes, y los ángulos de las rectas formadas por las trayectorias de cada paso del paciente. Para la pendiente se implementó la función

$$
M_{j}=\left(y_{i}^{\prime \prime}-y_{i-1}^{\prime \prime}\right) /\left(x_{i}^{\prime \prime}-x_{i-1}^{\prime \prime}\right) .
$$

$M$ es un vector de pendientes, donde las pendientes representan la razón de cambio de las mediciones, pudiendo diferenciar de esta manera los tipos de movimientos de los pacientes.

Los datos a utilizar corresponden al vector $S^{\prime \prime}$, creando un nuevo vector que llamaremos $S^{\prime \prime \prime}$, donde $s_{j}^{\prime \prime \prime}=M_{j} \mid 1 \leq j \leq n-1$. Por lo tanto, se tiene que

$$
S^{\prime \prime \prime}=\left\{s_{j}^{\prime \prime \prime}=M_{j}|1 \leq j \leq n| j, n \in \mathbb{N}\right\}
$$

Se define como un episodio de deambulaje al movimiento total realizado por un sujeto al perder la capacidad de reconocer el lugar o las personas que lo rodean, ya sea parcial o totalmente, además se conocerá a dicho episodio como $\Omega$.

\subsection{Módulo de clasificación de rutas}

Para el proceso de clasificación de la ruta obtenida (el conjunto de ángulos representa la ruta del sujeto), se utiliza la definición de los segmentos relevantes, donde se define como un segmento relevante a un conjunto de ángulos del mismo signo matemático. Se define también el concepto de segmentos no relevantes, que están formados por un máximo de dos mediciones de signo diferente al del resto de mediciones del segmento. Los segmentos no relevantes son causados por datos espurios que no fueron eliminados por los filtros aplicados a las mediciones obtenidas por los sensores.

Un segmento es un conjunto de mediciones del mismo tipo de datos (positivos o negativos) consecutivos, que pertenecen a la ruta definida con anterioridad. Estos segmentos nos arrojan información sobre el tipo de ruta seguida por el sujeto.

Se definirá $Z$ como un segmento, el cual está constituido por $n$ elementos positivos o negativos, pero no ambos. Se define entonces:

$$
\left.\mathrm{Z}=\left|m_{i}, m_{i+1}, \ldots, m_{i+n}\right| i, n \in \mathbb{N}\right\}
$$

Por la definición anterior, es correcto decir que un segmento relevante cumple ciertas condiciones, como son el numero de elementos que lo conforman y la consideración del punto donde los elementos del segmento realizan un cambio 
de razón, cambiando su signo e iniciando un nuevo segmento. Se dice que, un segmento relevante $Z^{\prime}$ esta formado por:

$$
Z^{\prime}=\left\{Z^{\prime} \subset \Omega\left|Z^{\prime}=\left[a<m_{i}, m_{i+1}, \ldots, m_{i+n}>b\right]\right| a, b, i, n \in \mathbb{N} \mid a \neq b\right\},
$$

donde $a, b$ son los límites que dictan la razón de cambio de los valores del segmento; es decir, si se supera dicho limite termina el segmento relevante.

Tipos de movimientos Los movimientos directos de acuerdo a [15], son movimientos realizados por el paciente cuando se dirige de un punto A a un punto B sin ningún tipo de desviación. Este tipo de movimiento está conformado por un solo movimiento relevante, ya sea de datos positivos o negativos,. De existir segmentos no relevantes, son ignorados en éste y todos los movimientos:

$$
M D=\left\{Z_{1}^{\prime} \subset \Omega\left|Z^{\prime}=\left[a<m_{i}, m_{i+1}, \ldots, m_{i+n}>b\right]\right| a \neq b \mid a, b, i, n \in \mathbb{N}\right\} .
$$

Los movimientos cíclicos son aquellos movimientos que realiza el paciente cuando se dirige de un punto $\mathrm{A}$ a un punto $\mathrm{B}$, después a un punto $\mathrm{C}$ y por ultimo a un punto D, regresando de vuelta al punto A. Este movimiento representa un giro en la ruta del paciente, de ahí el nombre de movimiento cíclico. Este tipo de movimiento está conformado por al menos cuatro segmentos relevantes, donde cada uno cuanta con más de cuatro mediciones, variando de datos positivos a datos negativos, además se puede identificar que el primer segmento relevante y el ultimo, generalmente son contrarios:

$$
\begin{aligned}
M C= & \left\{\left[Z_{1}^{\prime}, Z_{2}^{\prime}, \ldots, Z_{n}^{\prime}\right] \subset \Omega \mid Z^{\prime}=\right. \\
& {\left.\left[a<m_{i}, m_{i+1}, \ldots, m_{i+n}>b\right]|a \neq b| a, b, i, n \in \mathbb{N} \mid N \geq 4\right\} . }
\end{aligned}
$$

Los movimientos rítmicos son aquellos donde el paciente se mueve de un punto A a un punto $\mathrm{B}$ y después a un punto $\mathrm{C}$, repitiendo el mismo movimiento de manera consecutiva hasta llegar al objetivo. Este movimiento caracteriza por la existencia de al menor tres segmentos relevantes. Además, el primer segmento relevante es del mismo signo del último segmento relevante. Otra característica de este movimiento es que cuenta en cada segmento relevante con un valor máximo o un valor mínimo, donde los valores antes y después de este dato relevante, disminuyen o aumentan hasta cambiar de signo. Es decir, si tenemos un máximo, los valores antes y después de este disminuirán hasta cambiar de signo. Lo mismo ocurre con un mínimo; en este caso aumentarán hasta cambiar de signo.

$$
\begin{aligned}
M R= & \left\{\left[Z_{1}^{\prime}, Z_{2}^{\prime}, \ldots, Z_{n}^{\prime}\right] \subset \Omega \mid Z^{\prime}=\right. \\
& {\left.\left[a<m_{i}, m_{i+1}, \ldots, m_{i+n}>b\right]|a \neq b| a, b, i, n \in \mathbb{N} \mid N \geq 3\right\} . }
\end{aligned}
$$

Por último, los movimientos aleatorios son aquellos donde no existe un orden claro en la forma de movimiento del paciente, volviéndose en pocas palabras impredecible. Por lo tanto, cualquier movimiento que no cumpliera con las definiciones anteriores se considera como movimiento aleatorio. 


\subsection{Módulo analizador de rutas}

Este último módulo del componente presentado en este artículo se encarga de recopilar la información de los módulos anteriores y darles un trato de tal manera que el encargado o especialistas pueda interpretar la información correctamente. En este módulo se recopilará la información y se enviará al encargado/especialista, quien podrá verificar los resultados, filtrando la información por día, semana o mes, pudiendo llevar un control del paciente y realizar diagnósticos o tratamientos.

\section{Evaluación de la propuesta}

Para la evaluación de la propuesta se decidió utilizar un dispositivo similar al utilizado durante las pruebas realizadas por [9]. Dicho dispositivo es un dispositivo móvil, modelo «Samsung Galaxy s7». Al utilizar un dispositivo parecido en ambos casos se tuvo la ventaja de poder replicar los experimentos realizados en su trabajo previo, además de llevar a cabo pruebas en ambientes distintos.

Este dispositivo cuenta con varios sensores con los que se recolectaron los datos de los movimientos del sujeto. Por ejemplo, para poder recolectar información de la aceleración del sujeto se utilizó el sensor «K6DS3TR». Para obtener el ángulo de inclinación del sujeto con respecto al eje magnético terrestre, se utilizó el giroscopio «K6DS3TR». Para obtener la intensidad del campo magnético terrestre en la localización actual del dispositivo, se utilizó el sensor «YAS537».

Para probar la eficacia del sistema se llevaron a cabo una serie de experimentos donde se eligieron rutas al azar, juntando una muestra de 30 movimientos, de los cuales 10 fueron movimientos directos (D), 10 fueron cíclicos (C), 6 fueron rítmicos $(\mathrm{R})$ y 4 fueron aleatorios (A). Los resultados resultados de los experimentos se muestran en el Cuadro 3.

Tabla 3. Resultados de los experimentos.

\begin{tabular}{|c|c|c|c|c|}
\hline Movimientos $\mathrm{D}$ & $(\mathrm{N}=10)$ & $\mathrm{A}(\mathrm{N}=4)$ & $R(N=6)$ & $C(N=10)$ \\
\hline Exitos & 9 & 4 & 5 & 8 \\
\hline Fallos & 1 & 0 & 1 & 2 \\
\hline
\end{tabular}

Como se puede apreciar, el sistema obtuvo un error del $13.33 \%$ en las mediciones, donde los mayores problemas identificados, son las rutas rítmicas y cíclicas, esto debido a que ambos movimientos se asemejan al momento de aplicar la definición de los segmentos relevantes, pero a pesar de ellos, los resultados obtenidos son satisfactorios, esto debido a que se obtuvo una tasa de error similar o menor a la reportada por otras propuestas, con la ventaja de que la presentada en este artículo no requiere infraestructura previa y funciona tanto en interiores como en exteriores. 


\section{Conclusiones y trabajos futuros}

La demencia es uno de los principales problemas de salud actuales, debido en gran parte al envejecimiento de la población mundial. La demencia no tiene cura, provoca la muerte de quienes la padecen y son la causa de deterioro económico y emocional de familiares. Para dar el trato adecuado a una persona afectada con este padecimiento es necesario llevar a cabo un diagnostico preciso de la etapa de desarrollo de la enfermedad. Sin embargo, para determinarla es necesario llevar a cabo pruebas costosas y que provocan mucho estrés en los pacientes.

Recientemente se ha detectado que existe una relación entre los patrones de deambulaje y el nivel del avance de la demencia. En este artículo se presentó un componente para la detección de patrones de deambulaje de pacientes afectados con demencia. El componente propuesto, desarrollado con base en el paradigma del Internet de las Cosas, no es invasivo ni requiere de infraestructura previamente instalada en los edificios en los que será utilizado. La evaluación del sistema propuesta mostraron una alta tasa de detección.

Como trabajo futuro se tiene contemplado la integración de técnicas de aprendizaje autónomo (machine learning) en el módulo analizador de rutas.

\section{Referencias}

1. Algase, D.L., Antonakos, C.L., Beattie, E., Beel-Bates, C.A., Yao, L.: New parameters for daytime wandering. Research in Gerontological Nursing 2(1), 58-68 (jan 2009)

2. Alzheimer's Association: Types of Dementia. Alzheimer's Association (2017), https://www.alz.org/dementia/types-of-dementia.asp

3. Alzheimer's Association: Wandering and Getting Lost. Alzheimer's Association (2017), https://www.alz.org/care/alzheimers-dementia-wandering.asp

4. Alzheimer's Association: What Is Dementia? Alzheimer's Association (2017), https://www.alz.org/what-is-dementia.asp

5. Alzheimer's Association National Office: Alzheimer's Association National Office. Alzheimer's Association National Office (2017), http://www.alz.org/facts/overview.asp

6. Atzori, L., Iera, A., Morabito, G.: The internet of things: A survey. Computer Networks 54(15), 2787-2805 (2010), http://www.sciencedirect.com/science/article/pii/S1389128610001568

7. Bates, R.J., Bates, R.J.B.: GPRS: General Packet Radio Service (Professional Telecom). McGraw-Hill Professional (2001)

8. Budson, A.E., Solomon, P.R.: Memory Loss, Alzheimer's Disease, and Dementia: A Practical Guide for Clinicians. Elsevier (2015)

9. Camberos, M.L.L., Diaz-Ramirez, A., Marquez-Barja, J., Quintero, V.: Algoritmo de trazado de rutas en interiores sin infraestructura. In: Proceedings of International Conference on Computer Networks Applications. pp. 64-69. Tecnologico Nacional de Mexico (2017)

10. Chan, M., Bonhomme, S., Estève, D., Campo, E.: Individual movement trajectories in smart homes. In: IFMBE Proceedings, pp. 1014-1018. Springer Berlin Heidelberg (2008) 
11. Gavrilovska, L., et al.: Application and Multidisciplinary Aspects of Wireless Sensor Networks: Concepts, Integration, and Case Studies (Computer Communications and Networks). Springer (2010)

12. Google: Google Maps. Google (2018), https://cloud.google.com/maps-platform/

13. Kaplan, E., Hegarty, C.J.: Understanding GPS/GNSS: Principles and Applications, Third Edition (Gnss Technology and Applications Series). Artech House (2017)

14. Lin, Q., Zhang, D., Huang, X., Ni, H., Zhou, X.: Detecting wandering behavior based on GPS traces for elders with dementia. In: 2012 12th International Conference on Control Automation Robotics Vision (ICARCV). pp. 672-677 (Dec 2012)

15. Martino-Saltzman, D., Blasch, B.B., Morris, R.D., McNeal, L.W.: Travel behavior of nursing home residents perceived as wanderers and nonwanderers. The Gerontologist 31(5), 666-672 (oct 1991)

16. Mouly, M., Pautet, M.B.: The GSM System for Mobile Communications. Telecom Publishing (1992)

17. Organizacion Mundial de la Salud: Indice de Muertes por Demencia. Organizacion Mundial de la Salud (2009), http://www.who.int/gho/en/

18. Power, G.A.: Dementia beyond Disease: Enhancing Well-Being. Health Professions Press (2016)

19. Rodrigues, A., Silva, J.S., Boavida, F.: iSenior-a support system for elderly citizens. IEEE Transactions on Emerging Topics in Computing 1(2), 207-217 (dec 2013)

20. Sato, H.: Moving average filter (US patent US6304133B1) (2001)

21. Sposaro, F., Danielson, J., Tyson, G.: iWander: An Android application for dementia patients. In: 2010 Annual International Conference of the IEEE Engineering in Medicine and Biology. IEEE (aug 2010)

22. Vuong, N.K., Goh, S.G.A., Chan, S., Lau, C.T.: A mobile-health application to detect wandering patterns of elderly people in home environment. In: 2013 35th Annual International Conference of the IEEE Engineering in Medicine and Biology Society (EMBC). IEEE (jul 2013) 